\title{
Éditorial : une revue canadienne au rayonnement international
}

Cela fait plus de 30 ans que j'ai développé une affinité particulière avec le Canadian Journal on Aging /La Revue canadienne du vieillissement, soit depuis la publication de mon premier article dans le premier numéro de la revue. Je ne commencerai pas ma carrière éditoriale à la CJA/RCV avec une autocitation, mais vous pourrez toujours retrouver l'article dans les archives de la revue.

Accepter la fonction de rédacteur en chef de la $C J A / R C V$ représente un défi à la fois enthousiasmant et intimidant. Mon enthousiasme vient de la possibilité que j'aurai de soutenir et de poursuivre la fière tradition d'érudition de cette revue de gérontologie. Le défi est néanmoins intimidant et je suis conscient de la complexité et de l'importance des différents rôles qui sont en jeu - allant de l'apprentissage de la navigation sur le système de soumission en ligne, à l'évaluation du mérite d'articles de recherche couvrant une gamme de perspectives méthodologiques et disciplinaires.

La $C J A / R C V$ a eu la chance d'être servie par des équipes de rédaction et des rédacteurs en chef exceptionnels. Je suis donc reconnaissant de l'occasion qui m'est donnée de continuer à bâtir sur des bases aussi solides. Je tiens à remercier particulièrement ma prédécesseure immédiate, Dr Margaret Penning, pour son soutien et ses précieux conseils. Je voudrais également remercier Carlene Brennan pour tout son soutien pendant le processus de transition.

Je note que depuis que le Dr Penning a rendu son rapport annuel à l'assemblée annuelle de l'Association canadienne de gérontologie en 2014, le comité de rédaction de la revue a été complètement renouvelé. Je tiens à exprimer ma reconnaissance envers le Dr Penning et les autres éditeurs sortants pour leurs efforts dévoués, dont Dr Michel Bédard, rédacteur en chef adjoint; Dr Laura Funk, éditrice des comptes rendus de livre; Dr Nicole Caza, pour la psychologie; Dr Maureen Markle-Reid, pour les politiques sociales et la pratique; Dr Barbara Mitchell, pour les sciences sociales; et Dr Cara Tannenbaum pour la santé et les sciences biologiques. Je suis impatient de travailler avec les nouveaux membres de l'équipe éditoriale: Dr Sylvie Belleville, rédactrice en chef adjointe; Dr Kenneth Rockwood, éditeur des comptes rendus de livre; Dr Martine Lagacé, pour la psychologie; Dr Véronique Boscart, pour les politiques sociales et la pratique; Dr Deborah van den Hoonard, pour les sciences sociales; et Dr George Heckman, pour la santé et les sciences biologiques.
La $C J A / R C V$ est une revue canadienne ayant une portée internationale. Au cours des dernières années, la revue a reçu des manuscrits provenant de plus de 40 pays. La CJA/RCV compte plus de 7500 abonnés institutionnels, sur plusieurs continents. La revue, est distribuée à près de 5000 institutions dans les pays en voie de développement par les presses universitaires de Cambridge, grâce au programme HINARI de l'Organisation mondiale de la santé. Malgré ces statistiques impressionnantes, je crois que plusieurs opportunités pourront être saisies pour faire croître la revue sur le plan international.

Je ne crois pas que le «facteur d'impact » d'une revue soit toujours un indicateur valable de son importance. La $C J A / R C V$ est le plus important périodique en gérontologie au Canada et l'un des plus importants au monde. Il est donc clair que son influence et son importance dépassent largement ce que son facteur d'impact suggère. Cela étant dit, je souhaiterais voir le facteur d'impact de la $C J A / R C V$ augmenter dans les années à venir.

La $C J A / R C V$ fournit un espace propice à la publication d'un corpus extrêmement diversifié de travaux savants, ce que le numéro actuel illustre d'ailleurs très clairement.

Ce numéro comprend deux revues de littérature sur les services et les systèmes de santé. Un examen de la portée réalisée par Guruge, Thomson et Seifi, met en évidence les inégalités et les obstacles auxquels les immigrants âgés se heurtent lorsqu'ils veulent avoir recours à des services de santé mentale. Cette étude identifie aussi un certain nombre de priorités pour les recherches futures dans ce domaine. Tourigny et ses collègues rapportent les résultats d'un examen systématique des revues systématiques concernant les visites préventives à domicile - cet examen a été entrepris afin de conseiller le ministère de la Santé et des Services sociaux du Québec sur l'efficacité des programmes de soins à domicile. Malgré une richesse apparente des données empiriques disponibles, -10 revues systématiques antérieures ayant été identifiées - les auteurs ont trouvé que ces données étaient insuffisantes et ne permettaient pas de tirer des conclusions définitives, favorables ou non à ces programmes. Les questions posées par les auteurs seront familières à ceux qui ont suivi les travaux portant sur l'efficacité des services gériatriques multidimensionnels: quels sont les éléments clés de tels programmes et qui est le plus susceptible d'en bénéficier ? Une troisième question pourrait également 
être posée: quelles attentes raisonnables peut-on avoir par rapport aux résultats de ces programmes?

Dans leur article, McGregor et ses collègues ont abordé un sujet différent, mais leur travail laisse aussi présager des conséquences importantes pour le système de santé. Ils ont analysé des données recueillies à l'aide du RAI-MDS 2.0 dans les établissements de soins de longue durée en Saskatchewan et ont cherché à savoir si la couverture infirmière $24 / 7$ dans ces établissements signifiait une meilleure qualité de soins, telle que mesurée par le recours aux services de soins aigus. Leurs résultats suggèrent que cela pourrait être le cas. Pour autant, l'interprétation prudente de ces résultats reflète la complexité et les défis reliés à ce genre d'analyse.

L'article de McRae, d'une approche interactionniste et symbolique, explore la perspective des femmes âgées dans leurs interactions avec leur médecin et indique que la personnalité du médecin et «son attitude au chevet du patient» importent plus que ses compétences techniques ou son aptitude.

Deux articles abordent plusieurs aspects associés aux technologies et aux appareils d'assistance. Les systèmes intelligents de surveillance à domicile suscitent un intérêt croissant pour minimiser les risques et améliorer l'autonomie des personnes âgées. Cependant, cet intérêt s'accompagne de préoccupations légitimes: seront-elles bien reçues ou seront-elles plutôt perçues comme une intrusion non désirée? Ces questions feront partie des domaines de recherche explorés par le programme AGE-WELL, récemment financé des Réseaux de centres d'excellence (www.agewell-nce.ca). Dans ce numéro, Lapierre et ses collègues exposent une étude à méthodologie mixte qui montre que les soignants seraient prêts à utiliser un système de vidéosurveillance des chutes, malgré les préoccupations associées à la vie privée et au coût. Yip et ses collègues, quant à eux, présentent les résultats d'une revue de la littérature dont le but est de générer des recommandations pour la conception inclusive de rampes d'accès.

Deux autres articles portent sur des thématiques reliées au vieillissement des fonctions cognitives. Young-Bernier et ses collègues ont étudié la relation complexe entre l'activité cholinergique et le temps de réaction. Ils ont constaté que la baisse d'activité cholinergique n'était pas significativement associée à la performance et suggèrent que le dysfonctionnement cholinergique pourrait n'avoir un impact notable sur l'attention que lorsqu'il est sévère. Giroux et Stibre ont, quant à eux, utilisé une étude Delphi afin de valider un outil pour guider la prise de décision dans l'évaluation de la compétence chez des personnes âgées souffrant de troubles cognitifs.

D'autres publications reflètent une variété de préoccupations gérontologiques. Cusson et ses collègues de l'Université de Sherbrooke ont trouvé, en utilisant les données de l'étude NuAge, que l'efficacité masticatoire n'était pas associée à l'apport de nutriments essentiels.

Nicińska et Kalbarczyk-Steclik présentent une analyse de survie ambitieuse et complexe évaluant l'impact des événements marquants de la vie sur le risque de décčs chez les Européens âgés de 50 ans et plus. Bien que leurs résultats indiquent que ces événements influencent le risque de décčs, certaines conclusions sont surprenantes et méritent d'ętre approfondies. Par exemple, ils ont trouvé qu'une mauvaise santé durant l'enfance était associée ŕ un risque de décčs moins élevé.

La conduite automobile chez les personnes âgées fera l'objet d'un numéro spécial en 2016; dans ce numéro spécial, l'article de Johnston, Borkenhagen et Scialfa montre qu'il est important de faire une évaluation rigoureuse des différentes méthodes pour améliorer ou évaluer les capacités de conduite automobile des personnes âgées.

Enfin, ce numéro contient deux comptes rendus de livres. Fernandez a revu les essais rédigés à la suite des Rencontres de 2010 à Bad Aussee (Autriche). Ces derniers examinaient les représentations du vieillissement dans les médias, l'art et la littérature, en adoptant la perspective des études culturelles. Martin, quant à lui, présente le commentaire éloquent de Gawande sur l'incapacité du système de soins de santé à faire face au vieillissement et à la mort.

Je vous encourage à poursuivre vos activités en appui au CJA/RCV: envoyez-nous vos meilleurs travaux, participez au processus de révision et partagez vos idées afin d'améliorer la revue. Aussi, vous pouvez nous suivre sur Twitter : @cjarcv.

Paul Stolee

Rédacteur en chef 